\title{
PRESENTACIÓN
}

\section{EDUCACIÓN Y TRABAJO EN TIEMPOS DE TRANSICIONES INCIERTAS}

\author{
CLAUDIA JACINTO
}

Existe una larga tradición académica latinoamericana en investigaciones sobre educación y trabajo, basada en estudios que relacionan las credenciales educativas con la inserción ocupacional de los trabajadores, especialmente con sus ingresos y sus categorías socio-profesionales. Muchos trabajos se referenciaron en teorías del capital humano, al buscar relaciones entre educación y empleos o entre niveles de escolaridad y desarrollo.

Ahora bien, las vinculaciones lineales entre educación y trabajo vienen siendo largamente complejizadas y discutidas, tanto que la autora francesa Lucie Tanguy (1986) caracterizó el vínculo como "una relación inencontrable". Como sostiene De Ibarrola en su artículo en este dossier, los límites a la perspectiva optimista en términos de la movilidad asociada a los años de escolaridad aparecieron tanto desde las teorías críticas -que sugerían la reproducción continua de la sociedad, el sistema de dominación capitalista y la penetración de las desigualdades socioeconómicas más allá de la escolaridad-, como desde los trabajos que, profundizando desde la economía, el valor de los años de educación y su relación con los ingresos, mostraron cómo disminuía su valor como credencial.

En efecto, desde hace tiempo los jóvenes más educados tienden a desplazar a los menos educados, aun en empleos que no parecen demandar altas calificaciones técnicas, ante la escasez de empleos disponibles para todos (efecto fila). Estudios de demanda laboral también han detectado que suele requerirse educación secundaria como señal de contar con ciertas competencias generales, 
así como cierta presunción de comportamiento y disciplina, mucho más que por cierta calificación técnica.

En el caso de nuestra región, dos conceptualizaciones han iluminado este cuestionamiento a la linealidad de la adecuación entre niveles educativos, oferta formativa e inserción laboral planteada en las primeras versiones de la teoría del capital humano.

Desde el lado de las miradas sobre la educación, se ha evidenciado que su expansión se produjo con un grado creciente de segmentación, que configuró circuitos educativos de desigual calidad según los orígenes socioeconómicos y los capitales culturales de los hogares de los estudiantes, sumados a las diferencias y diversidades étnicas, de raza y de género. La baja calidad de la educación a la que acceden los niños y jóvenes provenientes de hogares con bajos ingresos también configura desventajas ante la inserción laboral. Esto enfrenta a los pobres que logran terminar el nivel secundario ante el sentimiento de que el esfuerzo educativo no se condice con las oportunidades que encuentran en el mercado laboral (Jacinto y otros, 2007).

Desde el lado de las miradas sobre el mundo del trabajo, el concepto de heterogeneidad estructural, introducido por la CEPAL casi desde sus orígenes, se refiere a la polarización dentro de las estructuras productivas y entre los sectores económicos: grandes diferencias de productividad del trabajo entre sectores y dentro de ellos, con innovaciones tecnológicas que se difunden lenta y desigualmente. La perspectiva de la heterogeneidad estructural incorporó luego nuevas dimensiones más allá de la divergencia tecnológica y de productividad, incluidas las desigualdades de género, los desequilibrios territoriales y la dimensión ambiental. De este modo, quedó ampliamente reconocido que los empleos disponibles también eran y son desiguales, heterogéneos y segmentados, y no podía esperarse que la educación fuera el vehículo unilineal de la movilidad social.

A lo largo de las décadas persiste una evidencia en la región: la concentración de la mayor parte del empleo en las actividades de muy baja 
Educación y Trabajo en tiempos de transiciones inciertas.

productividad y salarios (actividades informales o de subsistencia), que es una fuente de persistente desigualdad (CEPAL, 2016).

En resumen, la segmentación de los mercados de trabajo y los procesos de devaluación de credenciales educativas aparecen entre los grandes límites de la potencialidad de la educación y la capacitación para influir positivamente en las transiciones laborales de los jóvenes. La pregunta que nos formuláramos hace más de una década (¿Para qué trabajo formar?) sigue vigente a pesar de la expansión educativa y de las nuevas tecnologías de la información y comunicación. Planteábamos por esos años:

En América Latina, conviven sectores de punta integrados al mercado global, con diversos niveles tecnológicos, y un sector informal en el que trabajan los sectores más pobres de la población, que son mayoritarios. Una parte del creciente sector de servicios también se halla asociada al sector moderno, especialmente las comunicaciones y las nuevas tecnologías informáticas, implicando la necesidad del desarrollo de competencias transversales para toda la población. Ante esta compleja realidad existe un gran consenso acerca del valor de la educación y de la formación para la vida y el trabajo y sobre su aporte al desarrollo socioeconómico. Sin embargo, se enfrentan muchos interrogantes sobre hacia dónde debe orientarse una educación que amplíe las oportunidades de bienestar para todos los latinoamericanos. Entre ellos: ¿para qué mundo del trabajo formar?, ¿cómo promover alternativas educativas y laborales que permitan la inclusión de amplios sectores sociales hoy al margen de un trabajo decente?, ¿cómo promover redes de educación y trabajo vinculadas al desarrollo social?, ¿cuáles son las formas en las que se insertan en el trabajo los jóvenes egresados?, ¿qué puede hacerse desde la educación para un mejoramiento en las oportunidades de la inserción? (Jacinto, 2004, p. 12). 
Educación y Trabajo en tiempos de transiciones inciertas.

Como contribución sustantiva a este debate, De Ibarrola examina en su artículo los hallazgos de varias de sus investigaciones en pos de desentrañar las relaciones no lineales entre educación y trabajo, al analizar poblaciones con distintos niveles de escolaridad, en distintos sectores laborales, zonas geográficas de México y temporalidades. Partiendo desde lo escolar hacia lo laboral, sus investigaciones han conducido a una gran diversidad de espacios laborales, lo que descarta el supuesto de que un mercado de trabajo homogéneo opera con la misma racionalidad y los mismos mecanismos para toda la población demandante de trabajo.

De Ibarrola propone entonces la existencia de una estructura laboral heterogénea, desigual y combinada -que posteriormente se calificó como una matriz de heterogeneidad laboral-, basada en el rejuego de los indicadores de tres grandes criterios: el sentido/orientación del trabajo, la racionalidad que lo guía: acumulación capitalista, empleo público y trabajo de subsistencia; el grado de formalidad/informalidad de las relaciones laborales; la complejidad tecnológica y administrativa de las empresas. De este modo, concluye que escolaridad y empleo tienen derroteros diferentes según sectores - trabajadores con la misma escolaridad superior tienen brechas de ingresos importantes-, e incluso las brechas tendieron a reducirse entre los niveles de escolaridad a lo largo del tiempo.

Por otro lado, en los últimos años se destacan otras líneas de investigación en educación y trabajo que no son completamente nuevas, pero que han sido resignificadas y reorientadas en vinculación con los nuevos fenómenos y con los diálogos e interfaces que se vienen produciendo entre la sociología de la educación y los aportes de otras sociologías específicas, como la del trabajo y la de la juventud. Así, en un estudio anterior (Jacinto, 2011) se ha detectado un nuevo énfasis en las investigaciones, desde hace una quincena de años, que aborda las instituciones como el lugar donde se construye la implementación de los programas o políticas (en este caso, de educación y trabajo) y las lógicas de acción de los sujetos. En estas líneas, las voces de los distintos actores 
Educación y Trabajo en tiempos de transiciones inciertas.

implicados en la acción social son relevadas y trianguladas, en términos metodológicos.

Los estudios institucionales ya tenían variados antecedentes en décadas anteriores, y en trabajos recogidos en la década actual se observa que aportaron especialmente a entender las lógicas conflictuales en las que los actores involucrados construyen la acción. Se trata de trabajos que han estudiado las políticas o programas no desde un modelo evaluativo, para examinar solo su efectividad como si fuera una "caja negra" sino para comprender "la construcción social" (Gallart, 2006).

Entre las propuestas analíticas al respecto se destaca la que el artículo de De Ibarrola propone al referirse al concepto de estructuras institucionales del currículum, que pone de manifiesto las distancias entre los planes de estudio y la "realidad curricular": espacios muy limitados y deficientes para la formación técnica de los jóvenes; escasa consistencia entre las diferentes estructuras, que empieza por la inadecuada formación de los profesores, el contrasentido de los tiempos y calendarios escolares, la ausencia de los espacios previstos para la producción conforme al modelo educativo, la ausencia de recursos materiales para ello, entre otros.

Otro eje de nuevas aportaciones ha sido el pasaje de los tradicionales estudios de seguimientos de egresados a abordajes más complejos, que se anclan en las transformaciones ocurridas en la construcción de biografías en tiempos de incertidumbre respecto a los mundos del trabajo a los que se incorporan los jóvenes. Si las conocidas correlaciones entre niveles de escolaridad y empleos han cambiado sustantivamente a la luz de grandes cambios en los modelos de acumulación, los mercados de trabajo y las formas de organización del trabajo, se comprende que el fin de las relaciones lineales haya debilitado los estudios de egresados basados en el supuesto de la adecuación educacióntrabajo.

En este marco, son los estudios de trayectorias educativo-laborales, en un sentido temporal y procesual más amplio, los que van ocupando el lugar para comprender más complejamente la trama de niveles macro-sociales, meso- 
institucionales, biográficos y subjetivos que permiten comprender cómo se construyen las trayectorias juveniles. Así, hemos propuesto en un trabajo anterior (Jacinto, 2010) que el análisis de trayectorias educativo-laborales constituye una perspectiva teórica y metodológica que permite registrar las secuencias en el marco de un proceso temporal. En décadas pasadas (e incluso en la actualidad), los estudios de seguimiento de egresados solían tomar como punto de partida la salida o culminación de los estudios (secundarios o universitarios) y analizar las continuidades y rupturas de las sucesiones de acontecimientos referidos a lo laboral (o lo educativo), tomando como punto de llegada el fin del periodo analizado. Esta perspectiva funcionaba razonablemente bien para prever el impacto de los títulos en un mundo en el que los jóvenes lograban acceder a cierto nivel de estabilidad en el empleo de un modo adecuado a sus formaciones.

Pero la complejización de los procesos de inserción laboral de los jóvenes implica la necesidad de redefinir estos modelos basados en las vidas lineales donde se sucedían ciclos previsibles. Estamos ante una reorganización social de los ciclos de vida, es decir, en las trayectorias escolares, de las trayectorias laborales (especialmente en los primeros tramos), en las movilidades en el curso de la vida activa y en el proceso de salida (Galland, 2011). Estos momentos clave de los procesos de socialización están en crisis desde el doble punto de vista de las instituciones y de los individuos (Castel, 1997; Dubar, 1991. En este marco, el valor de los estudios de trayectorias radica en la posibilidad de dar cuenta de procesos de inserción laboral heterogéneos y desiguales, aun ante las mismas condiciones estructurales, como efecto de las transformaciones económicas de esta etapa del capitalismo y del debilitamiento institucional que deja a los sujetos sin tramas de protección social (Pérez Islas, 2008). El enfoque de las trayectorias permite develar el interjuego entre voluntad personal (fundada en sentidos, significaciones, estrategias o elecciones) y los condicionantes estructurales, contextuales e institucionales que se retroalimentan permanentemente a lo largo de la vida.

En este marco, el artículo de Sepúlveda se adscribe a los enfoques que incorporan las perspectivas de los sujetos y sus experiencias, durante y después 
del paso por una experiencia formativa. Esta es una tendencia investigativa que puede ser muy fructífera, y ha sido poco explorada con anterioridad, para analizar la educación técnica en la región.

Es que la educación técnica fue, desde sus inicios, considerada como la modalidad educativa vinculada por excelencia a la formación para el trabajo en el nivel secundario. Era $-\mathrm{y}$ sigue siendo según algunas concepciones- en este nivel educativo donde más debates y polémicas se suscitaron en torno a su función en los actuales contextos, al entender que si no se adecúa a las demandas productivas, ¿para qué sirve? Sin embargo, algunos trabajos sobre escuela técnica o dispositivos específicos ya advierten que se está produciendo una reconfiguración de su tradicional rol, en el marco de la ruptura de los procesos de socialización laboral, que excede su finalidad de formar en competencias específicas y evidencia nuevos sentidos e influencias sobre las subjetividades y trayectorias en torno al trabajo (Jacinto y Dursi, 2010).

Dando un paso más, Sepúlveda enfatiza los argumentos en torno al aporte que pueden tener estos análisis de trayectorias para la toma de decisiones y la formulación y evaluación de políticas públicas. De este modo, se inscribe en la corriente de trabajos de investigación —detectada en un reciente estado del arte regional (Jacinto, 2011) - que postula que una determinada política o programa es construido por la interacción conflictiva entre el modelo (normativo, regulado) y las instituciones y los actores sociales que lo desarrollan. Ello se evidencia en una tensión permanente e inevitable entre la naturaleza técnica de la implementación de la política y las condiciones socio-políticas y culturales en que se desarrolla.

Desde estos supuestos, una línea de trabajos plantea incluso una redefinición de los modelos de evaluación de políticas públicas, que deberían ser contextualizados, analizados en sus condiciones de implementación, teniendo en cuenta las mediaciones en el nivel macro y la escuela, y —agrega Sepúlveda— en función de la experiencia que viven los sujetos hacia los cuales están dirigidas ${ }^{1}$.

\footnotetext{
${ }^{1}$ Uno de los aportes de Stephen Ball en el campo educativo señala que, a diferencia de una perspectiva lineal, resulta más adecuado considerar la política como un sistema cíclico, ya que es creada y recreada a lo largo del tiempo por los distintos actores que participan o inciden en esta.
} 
Al analizar las múltiples trayectorias de jóvenes egresados de la educación media técnica con esta perspectiva, Sepúlveda concluye que la experiencia se presenta en un "continuo educación-trabajo". Los estudiantes se ven impulsados de manera temprana a enfrentar un mercado laboral que con frecuencia no resulta funcional a los diseños curriculares construidos en el ámbito educativo. Los hallazgos de este autor refuerzan una perspectiva crítica de la educación técnica, que ha estado presente desde hace varios años en el debate internacional, sobre si tiene sentido orientar esta modalidad educativa únicamente a la inserción temprana al mundo del trabajo, como en el caso de Chile.

Finalmente, otro grupo de aportaciones innovadoras a los estudios sobre educación y trabajo se ubican en el campo del análisis de subjetividades y sus relaciones con los cambios sociales, culturales y socioeconómicos más amplios.

El papel de los sujetos, los sentidos subjetivos de la acción o de la comprensión de la experiencia han surgido como temáticas propias de la posmodernidad. Con un nuevo lugar en los estudios sobre las relaciones entre educación y trabajo aparecen los jóvenes, punta de lanza de grandes transformaciones sociales especialmente observables en los procesos de transición educativo-laboral. Como sostienen Ferraris, Roberti, Burgos y Pozzer en su artículo, la mutación de las normas de empleo hacia un modelo más flexible, inestable y precario está acompañada por el declive de las instituciones antaño centrales para la socialización —la familia, la escuela, el empleo y los sindicatos(Dubet, 2002) y de una transformación de las temporalidades sociales y biográficas, que destacan la incertidumbre (Beck, 1998). Los seres humanos se sumergen en una búsqueda constante por intentar encontrar "soluciones biográficas" a contradicciones sistémicas en un contexto inestable y cambiante (Beck, 1998).

Los procesos de individualización apelan cada vez más al desarrollo de intervenciones y políticas que apunten a las subjetividades, lo que da espacio a una nueva generación de políticas de inclusión educativa y laboral que podrían encuadrarse dentro de lo que se ha dado en llamar políticas de subjetividad. Cuando el trabajo sobre las subjetividades se convierte en estrategia de inclusión 
se proponen acciones con un alto grado de personalización y de acompañamiento, donde se propende al desarrollo de actitudes, valores, motivaciones, expectativas y recuperación de la autoestima, entre otras (Jacinto y Millenaar, 2009).

Puestos a examinar la intervención de dispositivos que apuntan a la construcción de subjetividades, Ferraris y otros llaman la atención sobre el hecho de que se genera un doble juego en el que los dispositivos de formación y empleo inciden sobre las subjetividades -en sus opiniones, acciones y prácticas-, al tiempo que los protagonistas negocian sobre y con estas estrategias de intervención.

De este modo, convergen con la perspectiva planteada más arriba acerca de la construcción social de las políticas y la necesidad de analizarlas en los niveles macro, meso y microsocial, para comprender esas intersecciones, recreaciones y distancias que van desde el discurso de los implementadores a los receptores, desde la letra de la normativa a su reinterpretación por parte de los actores.

¿De qué se trata esta intervención sobre las subjetividades? En muchos casos, se centra en lo que se ha dado en llamar mediar sobre las disposiciones. Por un lado, las disposiciones respecto a la escolarización (como bien muestran los estudios que tratan sobre las estrategias de las escuelas para mediar entre el ser joven y el ser estudiante, y motivar a la permanencia en la vida escolar). Por otro lado, en lo que concierne al foco de este dossier, mediar sobre las disposiciones en torno al trabajo significa en muchos casos apoyar el desarrollo de saberes y competencias transversales, consideradas claves para todo trabajo, que apuntan muchas veces a la resolución de problemas y a lo actitudinal (más recientemente rebautizado como socioemocional), así como a la capacidad de concebir un proyecto socio-ocupacional que promueva la reflexividad individual y tienda a construir soportes institucionales y colectivos.

En definitiva, la eclosión de las subjetividades se produce ante el desdibujamiento de los soportes sociales y del debilitamiento de los grandes motores de movilidad social del siglo veinte: el empleo y la educación. Y son estas políticas sectoriales las que están respondiendo, a través de dispositivos diversos, 
a lo que algunos ven críticamente como un tratamiento individualizado de la pobreza a una "ciudadanía en negativo", según la concepción de Castel (1997).

Analizando dispositivos de orientación ubicados en las políticas de empleo de jóvenes, y de educación de jóvenes y adultos en Argentina, el artículo de Ferraris y otros examina las contradicciones internas entre la letra y los procesos y espacios de implementación. Por ejemplo, si bien las prestaciones del programa de empleo juvenil se presentan como acciones integradas, las trayectorias que despliega cada uno de los participantes son particulares: luego de un módulo introductorio, los participantes desarrollan un trayecto más individualizado y se diseminan por un conjunto variado de instituciones que participan como prestadoras de los servicios de empleo, lo que dificulta una intervención personalizada. Esta debilidad en los procesos de acompañamiento se agudiza con la masificación del programa.

Ahora bien, en el marco del módulo introductorio del programa se pone en marcha una figura que desde hace algo más de una década también suele estar presente en las instituciones educativas (especialmente aquellas que trabajan en contextos difíciles y con riesgos de alto abandono escolar): la figura del tutor, del acompañante, de aquel con el que se generan relaciones de confianza que actúan como protectoras, facilitando el despliegue de las subjetividades a las que se apunta. En el caso de la institución educativa de jóvenes y adultos examinada, su carácter más institucionalizado y el compromiso de los docentes aportan una intervención más global, más permanente, que trata de intervenir en simultáneo sobre la formación de capacidades sociolaborales, el fortalecimiento de los aprendizajes y el empoderamiento de los sujetos.

Aunque los autores de este artículo aclaran que no pretenden comparar las experiencias que utilizan para examinar distintos modelos de intervención sobre las subjetividades, algunas diferencias saltan a la vista: mientras una es una institución con presencia territorial permanente, la otra responde a un programa con componentes diversos, siempre más "vulnerable" a los avatares de la implementación y de los cambios de política. Así, en la primera es la propia institución la que tiene una estrategia de tutoría que puede trascender a 
las personas; en cambio, en la segunda, la figura del tutor está centrada en una persona que tiene presencia dispar a lo largo del paso por el programa. La estrategia audaz del programa, de funcionar como articulador de servicios (interesante aprovechamiento de servicios de formación profesional, educación de adultos y prácticas laborales disponibles) encuentra su punto más débil en la precariedad del dispositivo de acompañamiento a lo largo de todo el paso por el programa.

Sin embargo, el problema en torno al acompañamiento está presente en los dos casos: la debilidad de las tramas de protección en las poblaciones vulnerables excede el paso por un programa o institución. Es una condición de la vida en contextos de precariedad en el estadio actual del capitalismo y, por ello, el acompañamiento de trayectorias precisa trascender los períodos y los pasos por programas e instituciones para enfrentar la inseguridad social, en términos de Castel, a lo largo de toda la vida.

En esta introducción se intentó resaltar de qué manera los trabajos incluidos en el dossier se insertan, al menos en una parte, en las tradiciones latinoamericanas de las investigaciones sobre educación y trabajo. Lo que resulta transversal a todas ellas es el reconocimiento de una necesaria multidimensionalidad en los análisis que vinculan ambos factores, tanto en términos espaciales, contextuales o temporales, como en la propia comprensión de las relaciones de top-bottom y bottom-up, entre los procesos macro-sociales y las políticas de educación, trabajo y protección social, las instituciones y los sujetos y sus trayectorias.

\section{REFERENCIAS}

Beck, U. (1998). La sociedad del riesgo. Buenos Aires: Paidós.

Dubar, C. (1991). La Socialisation. París: Armand Colin.

Dubet, F. (2002). Le déclin de l'institution. París: Seuil.

Castel, R. (1997). La metamorfosis de la cuestión social. Una crónica del salariado. Buenos Aires: Paidós. 
CEPAL. (2016). Horizontes 2030: La igualdad en el centro del desarrollo sostenible. Presentado en el $36^{\circ}$ periodo de sesiones. Ciudad de México, México.

Galland, O. (2011). La sociologie de la jeunesse. París: Armand Colin.

Gallart, M. A. (2006). La construcción social de la escuela media. Una aproximación institucional. Buenos Aires: La Crujía-Editorial Stella.

Jacinto, C. (Coord.). (2004). ¿Educar para que trabajo? Discutiendo rumbos en América Latina. Buenos Aires: redEtis (IIPE-IDES)-MTEySS-MECyT-La Crujía.

Jacinto, C., Longo, M. E., Bessega, C. y Wolf, M. (2007). Jóvenes, precariedades y sentidos del trabajo. Un estudio en Argentina. Revista Medio Ambiente y Urbanización, 66, 3-22.

Jacinto, C. (2010). Introducción. Elementos para un marco analítico de los dispositivos de inserción laboral de jóvenes y su incidencia en las trayectorias. Jacinto, C. (Comp.). La construcción social de las trayectorias laborales de jóvenes. Políticas, instituciones, dispositivos y subjetividades (pp. 15-49). Buenos Aires: Teseo-IDES.

Jacinto, C. y Millenaar, V. (2009). Enfoques de programas para la inclusión laboral de los jóvenes pobres: lo institucional como soporte subjetivo. CIDPA Última Década, 17 (30), 67-92.

Jacinto, C. (2011). Aproximaciones a un estado del conocimiento sobre formación para el trabajo en cinco países latinoamericanos. Tendencias en Foco, 20, 1-11.

Jacinto, C. y Dursi, C. (2010). Los nuevos sentidos de las pasantías en la escuela secundaria. Propuesta Educativa, 33, 85-93.

Pérez Islas, J. A. (2008). Entre la incertidumbre y el riesgo: ser y no ser, esa es la cuestión juvenil. Bendit, R., Hahn, M. y Miranda, A. (Coords.). Los jóvenes y el futuro. Procesos de inclusión y patrones de vulnerabilidad en un mundo globalizado (pp. 175-192). Buenos Aires: Prometeo.

Tanguy, L. (Dir). (1986). L'introuvable relation formation-emploi. París: La documentation française. 


\section{Claudia Jacinto}

Licenciada en Sociología (UBA), doctora en Estudios de América LatinaSociología (Universidad de París III). Coordinadora del PREJET (Programa de Estudios sobre Juventud, Educación y Trabajo) del Centro de Investigaciones Sociales (CIS-CONICET) del IDES. Investigadora principal del Consejo Nacional de Investigaciones Científicas y Técnicas de Argentina. Vice-presidente de la Asociación Latinoamericana de Estudios del Trabajo (ALAST). Docente de posgrado de la UBA, FLACSO y la Universidad Nacional del Comahue. Ha sido profesora invitada de posgrados en la Universidad Autónoma de Barcelona, Universidad de París $\mathrm{V}$ y la Universidad de la República de Uruguay. Se especializa en temas de educación secundaria y formación para el trabajo; políticas de formación profesional y empleo; juventud y transiciones educaciónformación-trabajo. 